\title{
LES SYNTAGMES NOMINAUX FRANÇAIS DANS UNE PERSPECTIVE VALENTIELLE
}

Devant la carence de la grammaire traditionnelle lorsqu'il s'agit de définir la diversité des liens pouvant exister au sein de syntagmes nominaux complexes (SN), nous proposons une classification des substantifs français et de leurs expansions, qui vise à une compréhension d'ensemble de ce type de constructions. ${ }^{1}$ A cette fin, les SN seront considérés comme représentant la transposition d'une proposition libre en forme nominale et comme reproduisant à ce titre le schéma distributionnel observable au niveau de la phrase. Nous appliquerons par conséquent, pour les examiner, les règles élaborées en matière de valence verbale.

Notre analyse s'inspire de la notion de valence telle qu'elle a été définie par Lucien Tesnière (1959) et de la distinction qu'il effectue entre les actants et les circonstants, la valence étant pour lui le nombre d'actants qu'un verbe est susceptible de régir, alors que les circonstants ne font qu'indiquer les circonstances de l'action.

Dans leur développement de cette théorie, Michael Herslund et Finn Sørensen (Herslund et Sørensen 1985, Herslund 1988a, 1988b, ce volume) opèrent non plus avec une seule mais avec deux distinctions fondamentales: d'abord une dissociation entre les membres de phrase déterminés par le contenu lexical du verbe ${ }^{2}-$ ce sont les expansions essentielles ou liées - et ceux qui sont ajoutés à la phrase entière - les expansions circonstancielles ou libres; ensuite, une division entre arguments et modifieurs, c'est-à-dire entre les membres de phrase qui dénotent les entités entre lesquelles sont établies les relations désignées par le verbe (leur fonction syntaxique est celle de compléments), et ceux qui qualifient ces relations en leur attribuant une propriété ou en leur associant une quantité (il s'agit ici d'adverbes ou de locutions adverbiales exprimant la manière, l'intention, la cause, la concession, la quantité, etc....).

Herslund et Sørensen aboutissent de la sorte au tableau suivant, dans lequel seuls les éléments de la catégorie a. déterminent la valence du verbe au sens de Tesnière. Ils

1 Pour une approche analogue des syntagmes nominaux danois, voir Baron (à paraître).

2 Qu'un membre de phrase soit 'déterminé' ou 'impliqué' par le contenu lexical d'un verbe signifie qu'on ne peut nier son existence sans nier en même temps l'acte verbal proprement dit (cf. Herslund et Sørensen 1985: 36 ss, Herslund 1988a: 29-34). Ex. il n'a rien vendu à personne = il n'a pas vendu, le contrat ne dure aucun temps = le contrat ne dure pas. 
comprennent le sujet, l'objet et l'adjet. Ce dernier recouvre l'objet prépositionnel, le complément d'attribution, le complément local et les attributs du sujet et de l'objet, chacun saturant à lui seul la place de l'adjet.
Expansions essentielles
Expansions circonstancielles
Arguments
a. complément essentiel
b. complément circonstanciel ou actant ou circonstant
Modifieurs
c. adverbe essentiel
d. adverbe circonstanciel

Tableau 1

Nous fondant sur ces définitions, nous poserons comme hypothèse de départ que le schéma ci-dessus peut être transposé aux syntagmes nominaux, ainsi qu'il ressort des exemples suivants:

(1) a. La vente des produits agricoles par le producteur

b. La vente des produits agricoles sur le marché

c. Un poids de dix kilos

d. Une conférence de deux heures

Nous nous limiterons à une analyse des expansions ayant la forme de syntagmes prépositionnels. ${ }^{3}$ Notre corpus se compose de $800 \mathrm{SN}$ répartis pour l'essentiel sur six contrats commerciaux. Le choix d'un corpus de textes juridiques tient au nombre particulièrement élevé de tournures nominales qu'on y rencontre (cf. Sourioux/Lerat 1975 et Cornu 1990). Ces tournures se présentent ici sous leur aspect canonique dans la mesure où le besoin de précision exclut les expressions imagées, métaphoriques et autres variations stylistiques des formes initiales.

La transposition immédiate du schéma verbal ci-dessus aux syntagmes nominaux n'est possible qu'avec les substantifs noyaux directement dérivés de verbes. La question se pose alors de savoir si une classification de ce type permet également d'analyser les substantifs non déverbaux, mais présentant néanmoins un schéma valentiel, ainsi que les noyaux ne pouvant en aucun cas s'associer un actant. Afin de répondre à cette question, nous proposons de répartir les substantifs en deux catégories selon qu'ils reproduisent ou non la valence des verbes, à savoir les substantifs valentiels et les substantifs non valentiels, et nous nous attacherons à définir la nature de la dimension verbale pouvant exister au sein d'un SN. Ceci devrait par contrecoup nous permettre de préciser la notion de valence appliquée aux constructions nominales. Pour ce faire, nous examinerons dans une première partie les substantifs valentiels et leurs compléments essentiels ou actantiels; puis, dans une seconde partie, nous délimiterons les expansions non actantielles de ces mêmes substantifs; dans une 
troisième partie enfin, nous verrons dans quelle mesure une classification similaire est applicable aux substantifs non valentiels.

\section{Les substantifs valentiels et leurs expansions actantielles}

Il convient de définir plus exactement la catégorie des substantifs valentiels, avant d'aborder l'étude de leurs expansions actantielles.

1.1 Les substantifs valentiels sont les substantifs qui établissent un rapport verbal avec une ou plusieurs de leurs expansions en reproduisant la valence des verbes ou des locutions verbales dont ils sont dérivés directement ou indirectement.

1.1.1 Les dérivés directs sont des noms dérivés de verbes pleins. Ils sont formés d'une part au moyen de dérivations régulières et productives à l'aide de suffixes comme -tion, -ation, -(s)sion, -aison, -ment, -ée.... évolution, acceptation, succession, livraison, placement, durée,... et d'autre part au moyen de dérivations irrégulières, non productives et sans marques morphologiques spécifiques comme facture, achat, désir, fin...

Ces dérivés directs se répartissent en trois sous-catégories suivant le type de nominalisation qu'ils expriment. Le cas le plus fréquent est celui de la transposition nominale du procès lui-même: nous qualifierons ce type de nominalisation nucléaire par opposition à deux autres sous-catégories possibles, à savoir la nominalisation subjective et la nominalisation objective désignant respectivement le sujet et l'objet, c'est-à-dire le résultat, de l'action exprimée par le verbe. ${ }^{4}$ C'est ainsi qu'à un même verbe transitif pourront correspondre les trois types de nominalisation, subjective, objective et nucléaire, suivants:

$\begin{array}{llll}\text { Verbe } & \text { NomSubj } & \text { NomNucl } & \text { NomObj } \\ \text { produire } & \text { producteur } & \text { production } & \text { produit } \\ \text { accuser } & \text { accusateur } & \text { accusation } & \text { accusé }\end{array}$

Précisons que tous les verbes n'auront pas toujours les trois possibilités de transposition; de plus, il pourra y avoir identité formelle entre les nominalisations nucléaire et objective:

$\begin{array}{llll}\text { facturer } & - & \text { facturation } & \text { facture } \\ \text { créer } & \text { créateur } & \begin{array}{l}\text { création } \\ \text { création }\end{array} \\ \text { livrer } & \text { livreur } & \text { livraison } & \text { livraison } \\ \text { acheter } & \text { acheteur } & \text { achat } & \text { achat }\end{array}$

La nature de la nominalisation va jouer un rôle décisif quant à la possibilité pour un substantif de régir des compléments actantiels. Ainsi, une nominalisation nucléaire

4 Cf. Tesnière (1959: 403 ss), Comrie/Thompson (1985: 357), Stage (1986: 211) et Ulland (1991: 4 ss). Nous exluons du cadre de notre étude les nominalisations locatives et instrumentales comme abattoir ou arrosoir. 
peut impliquer un ou plusieurs actants, selon la valence du verbe auquel elle correspond (ex. 'l'accusation de $X$ par $Y$ ', cf. ' $Y$ accuse $X$ '). Par contre, une nominalisation objective qui englobe, outre l'action verbale, un des actants - à savoir l'objet - ne pourra s'associer qu'un actant autre que celui qu'elle désigne, soit un sujet ou éventuellement un adjet (ex. 'la facture de M. Dubois', 'l'aide aux travailleurs immigrés'). Inversement, une nominalisation subjective, qui contient à la fois le verbe et son sujet, ne peut être suivie que d'un complément objectif (ex. 'les dirigeants de la société'). ${ }^{5}$ Les diverses expansions actantielles permettront par conséquent de désambiguïser, le cas échéant, des nominalisations nucléaire et objective homonymes. Ainsi, si livraison employé isolément est ambigu, le terme ne peut traduire qu'un procès et non un résultat dans la livraison des marchandises, puisqu'il y est suivi d'un objet. ${ }^{6}$ Mentionnons finalement que les transpositions nominales renfermant des actants seront généralement plus concrètes, et s'emploieront par conséquent plus aisément avec la marque du pluriel (ex. 'les achats de la société', où achats est une nominalisation objective suivie d'un sujet: la société, mais non *'les achats des marchandises', où marchandises serait objet). Elles pourront également se combiner avec des adjectifs ne pouvant qualifier que des phénomènes concrets (ex. 'une confirmation illisible').

\subsubsection{Les dérivés indirects comprennent deux séries d'occurrences:}

- les cas où le substantif noyau du SN est dérivé d'un adjectif qui peut figurer comme attribut du sujet après un verbe comme être:

(2) responsabilité (être responsable)

défectuosité (être défectueux)

solvabilité (être solvable)

- les cas où le substantif noyau correspond au nom contenu dans une construction à verbe support (CVS). Nous entendons par là les locutions verbales formées à l'aide de verbes plus ou moins vides de sens suivis d'un substantif avec ou sans déterminant. Le verbe support peut être soit avoir et des variantes comme prendre, remporter (variantes inchoatives) ou donner, rendre, porter, apporter (variantes causatives):

(3) droit (avoir droit à)

5 Nous n'avons pas rencontré d'exemples de nominalisation subjective combinée avec un adjet. Remarquons de plus qu'un petit groupe de verbes pourra faire l'objet, en particulier dans les textes juridiques, d'une transposition nominale sous forme d'adjet: léguer - légataire; destiner destinataire. Comme pour les autres types de nominalisation, le substantif ne s'associera qu'un actant différent de celui qu'il comporte, soit ici un sujet ou un objet (ex. 'le légataire de M. Dubois', 'le destinataire des marchandises').

6 D'autres procédés de désambiguïsation existent également qui excèdent le cadre de l'analyse interne des SN. Mentionnons pour mémoire les restrictions sélectionnelles du verbe de la phrase entière. Si ce verbe ne peut se construire qu'avec un sujet ou un objet concrets, il ne pourra pas régir une nominalisation nucléaire, qui est un procès et donc une entité abstraite. Ex. 'il a trouvé la livraison sur le port', mais non *'il trouvé la livraison des marchandises sur le port’. 


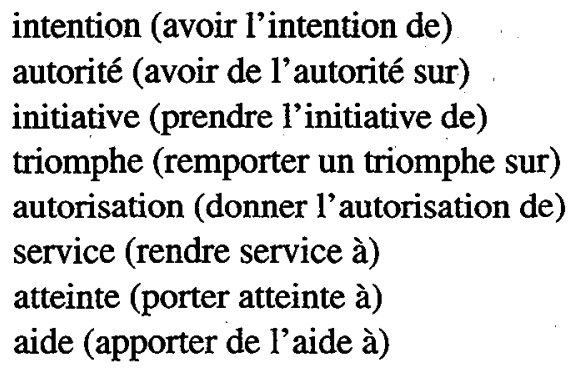

soit être ou faire et des verbes assimilés à faire comme fixer, commettre, écrire...: ${ }^{7}$

(4) guerre (être en guerre contre)

frais (faire les frais de)

prix/tarif (fixer le prix/le tarif de)

infraction (commettre une infraction à)

texte (écrire un texte sur)

On rencontre parfois des verbes transitifs coexistant avec une CVS (ex. atteindre porter atteinte à; aider - apporter de l'aide à; enfreindre - commettre une infraction a). ${ }^{8}$ Nous considérons dans de tels cas que le noyau d'un syntagme nominal est dérivé du substantif contenu dans la construction à verbe support et non du verbe correspondant. En effet, la préposition du SN est la même que celle de la CVS (ex. 'une atteinte à la liberté'/ ' $\mathrm{X}$ porte atteinte à la liberté', cf. 1.2.4), alors que le verbe n'est suivi d'aucune préposition (ex. 'atteindre'). On constate donc que l'évolution de la langue a eu comme point de départ le verbe, qui a été dérivé en substantif, lequel a été repris dans une construction à verbe support, avant d'être utilisé pour former un syntagme nominal. Plus généralement, on peut conclure qu'une CVS se distingue des autres combinaisons de forme verbe + objet en ce qu'elle peut être transformée en un SN. L'objet devient le noyau de ce syntagme et régit un adjet introduit par la même préposition que l'adjet de la locution verbale.

On voit, et il importe de le souligner, qu'il n'y a pas de parallélisme systématique entre la nature déverbale et le caractère valentiel des substantifs, puisqu'un nom, pour avoir des compléments actantiels, n'a pas besoin d'être dérivé d'un verbe (ex. droit).

\subsection{Les expansions actantielles}

Elles concordent avec les compléments essentiels de Herslund et Sørensen (cf. Tableau 1, a). Tout comme les actants définissaient la valence verbale au sein de la phrase, les expansions actantielles vont définir la valence du substantif au sein du SN. Elles s'expriment au moyen de syntagmes prépositionnels. La structure de ces constructions

7 Les verbes assimilés à faire ne sont considérés comme verbes supports que dans les cas où ils sont suivis du résultat de l'action qu'ils expriment, comme dans 'fixer le prix des marchandises', mais non dans 'fixer une carte sur un mur'.

8 Dans un nombre restreint de cas, ce sont des verbes intransitifs qui coexistent avec la CVS (ex. triompher de - remporter un triomphe sur). 
nominales peut être représentée sous la forme N1 prép N2, où N2 sera sujet (S), objet (O) ou adjet (A) de l'acte verbal contenu dans le noyau.

1.2.1 Les compléments subjectifs correspondent au sujet d'une proposition libre. La préposition employée sera typiquement de:

(5) la durée/l'expiration $d u$ contrat (le contrat dure/expire)

les désirs des clients (les clients désirent)

la confirmation préalable et écrite de la société (la société confirme préalablement et par écrit)

Accessoirement, la préposition employée est entre, si le nom valentiel dénote une relation entre deux sujets:

(6) la collaboration entre la société $X$ et la société $Y$ (la société $X$ et la société $Y$ collaborent)

1.2.2 Les compléments objectifs correspondent à l'objet d'une proposition libre. Lee cas canonique est ici aussi un syntagme introduit par la préposition de:

(7) la livraison des marchandises ( $\mathrm{X}$ livre les marchandises)

la rédaction $d u$ contrat en langue française ( $\mathrm{X}$ rédige le contrat en langue française)

le placement des articles suivants (X place les articles suivants)

l'exercice des fonctions telles que définies au présent contrat ( $\mathrm{X}$ exerce les fonctions telles que définies au présent contrat)

Cette similitude de forme entre le sujet et l'objet dans un syntagme nominal confirme ce que faisait déjà observer Emile Benveniste à propos du latin, à savoir que "l'opposition nominatif-accusatif, fondamentale dans le syntagme verbal, est neutralisée formellement et syntaxiquement dans le génitif déterminant nominal" (Benveniste 1966: 147). La distinction entre un sujet et un objet se situe par conséquent sur le plan logico-sémantique lorsque l'un des deux figure comme actant unique (ou éventuellement accompagné d'un adjet) d'un substantif valentiel.

1.2.3 Or, un même noyau, s'il s'agit de la nominalisation nucléaire d'un verbe transitif, peut régir simultanément les deux compléments subjectif et objectif. Dans ce cas, le sujet sera normalement introduit par la préposition par et l'objet par $d e:^{9}$

(8) la confirmation de la commande par la société (la société confirme la commande) l'acceptation des ordres par le mandant (le mandant accepte les ordres)

l'encaissement de la facture par le concédant (le concédant encaisse la facture)

le refus par l'agent d'accepter ces modifications (l'agent refuse d'accepter ces modifications) ${ }^{10}$

9 L'objet combiné avec un sujet en par peut également avoir la forme d'un adjectif possessif, en ou dont (cf. Wiberg 1956: 172 ss).

10 Lorsque l'objet est une construction infinitive, par alterne avec de. Ex. 'le refus du général de Gaulle de prendre le pouvoir' (cf. Spang-Hanssen 1963: 34). 
Parfois, l'objet est omis s'il ressort nettement du contexte:

(9) En cas de résiliation _ par l'une ou l'autre des parties, les commissions seront dues sur toutes les commandes provenant de la clientèle définie à l'article ler Le péril qu'avait fait courir à la France l'encerclement _ par l'Espagne et la Maison d'Autriche est définitivement écarté (cit. Wiberg 1956: 173) ${ }^{11}$

Soulignons que ces observations ne s'appliquent pas lorsque le noyau est dérivé d'un verbe de sentiment. Dans ce cas, c'est le sujet qui est introduit par de, tandis que l'objet est exprimé à l'aide de pour: 'l'amour d'Edouard pour sa mère' (Edouard aime sa mère).

1.2.4 Le substantif noyau peut être caractérisé finalement par un adjet sous forme d'un syntagme prépositionnel qui équivaut à différentes réalisations de la relation verbe/ locution verbale-adjet dans la proposition libre. A de rares exceptions près (cf. note 13), la préposition employée sera la même qu'après les verbes ou locutions verbales correspondantes. L'adjet peut être un objet prépositionnel 'neutre' ( $\mathrm{A}_{\text {neu }}$ ) introduit normalement par à ou $d e$ :

(10) le consentement du mandant à toute succession qui lui est présentée (le mandant consent à toute succession qui lui est présentée)

une atteinte à la liberté individuelle (X porte atteinte à la liberté individuelle) toute infraction à une règle de Droit international (X commet une infraction à une règle de Droit international)

son intention de renouveler le présent contrat (X a l'intention de renouveler le présent contrat)

L'adjet peut être également un complément datif $\left(A_{d a t}\right)$ introduit par $a$ :

(11) la vente des produits à la clientèle ( $\mathrm{X}$ vend les produits à la clientèle)

l'aide aux travailleurs immigrés ( $\mathrm{X}$ apporte de l'aide aux travailleurs immigrés)

ou bien un complément local $\left(\mathrm{A}_{10 c}\right)$ dans lequel le choix de la préposition varie avec la nature du lieu exprimé:

(12) une meilleure pénétration sur le marché danois (X pénètre mieux sur le marché danois)

l'extension de son champ d'activité sur de nouveaux articles ou de nouvelles marques (X étend son champ d'activité sur de nouveaux articles ou de nouvelles marques)

l'installation de téléphones chez les personnes âgées, cit. Stage, à paraître (X installe des téléphones chez les personnes âgées)

1.2.5 Les expansions actantielles des substantifs n'ont pas besoin de figurer expressément dans le syntagme. Elles peuvent très bien n'être présentes qu'à l'état latent. Ainsi dans les ventes directes sur le secteur, les $\mathrm{S}, \mathrm{O}$ et $\mathrm{A}$ sont tous sous-

11 Pour une analyse de ces constructions comme des manifestations de structures ergatives, voir Herslund (1982). 
entendus. Un verbe au contraire aura presque toujours besoin de la présence explicite de ses actants - au moins de son sujet - puisque sa fonction essentielle est de former une proposition.

Comme nous l'avons laissé entendre plus haut (cf. 1.2.3), les compléments actantiels peuvent se combiner entre eux à l'intérieur du même syntagme. Toutefois, nous n'avons pas rencontré d'exemples de SN comportant plus de deux actants, bien que ces cas soient théoriquement possibles. Le $S$ et l'O sont en général placés plus près du noyau que l'A, ainsi qu'il ressort des exemples (10), (11) et (12). ${ }^{12}$

Enfin, une expansion actantielle peut, à son tour, comporter un ou plusieurs compléments, situés en quelque sorte à un second ou à un troisième niveau de profondeur:

(13) la notification de la résiliation $(\mathrm{O})$ du présent contrat $(\mathrm{O})$

la concession exclusive de la vente $(\mathrm{O})$ de ses machines-outils $(\mathrm{O})$

une analyse de l'évolution $(\mathrm{O})$ des tarifs $(\mathrm{S})$ de la société $X(\mathrm{~S})$

Il est à noter ici que deux expansions introduites par de à l'intérieur du même SN sont seulement possibles dans les cas suivants:

- si elles sont situées à des niveaux de profondeur différents, comme dans les exemples ci-dessus,

- si une des expansions est un $\mathrm{A}_{\text {loc }}$ (ex. 'le retrait des troupes israéliennes des territoires occupés', cit. Herslund 1982: 78),

- ou si une des expansions est une construction infinitive objet (ex. 'le refus du général de Gaulle de prendre le pouvoir', cf. note 10) ou adjet (ex. 'la liberté des parents d'avoir ou de ne pas avoir des enfants', cit. Stage, à paraitre, 'l'intention d'une des parties de ne pas renouveler le présent contrat'). ${ }^{13}$

1.2.6 Mentionnons enfin une catégorie un peu spéciale formée de structures inversées, qui se rencontrent surtout dans les textes journalistiques et littéraires, où N1 est un complément essentiel d'un N2 déverbal:

(14) (S) l'homme de la russification [l'homme russifie] (cit. Bartning 1992: 170)

12 D'autres facteurs, telles la longueur des expansions et plus généralement la structure informationnelle dans le texte, influencent sans doute aussi l'ordre des compléments au sein du $\mathrm{SN}$. Nous reviendrons sur la question dans un article ultérieur.

13 Lorsque la construction infinitive est un adjet, de alterne dans certains cas avec à, même si l'adjet de la construction verbale correspondante ne peut être introduit que par la préposition de. Il en est ainsi par exemple de capacité et de incapacité. Ex. 'une incapacité des dirigeants à faire face aux problèmes du présent', cit. Stage, à paraître (mais: 'les dirigeants sont incapables de faire face aux problèmes du présent'). Voir à ce sujet Spang-Hanssen (1963: 107). Ajoutons que les syntagmes comportant des régimes indéterminés comme dans 'la volonté de puissance de certains politiciens' seront traités sous 2.3. Quant aux constructions du type la photo de mon fils de ta mère, elles relèvent davantage de la langue parlée et nous paraissent trop marginales pour être retenues dans cette étude. 
(O) la Pologne de l'invasion [X envahit la Pologne $]^{14}$

(Adat) les enfants de la promesse [X fait une promesse aux enfants] (Robert 1991: promesse)

Nous venons de décrire les compléments actantiels qui définissent la valence des substantifs. Il nous faut à présent classer les autres expansions possibles dans les SN comportant un noyau valentiel.

\section{Les substantifs valentiels et leurs expansions non actantielles}

Nous fondant toujours sur le tableau quadripartite de Herslund et Sørensen, nous analyserons successivement les compléments circonstanciels et les modifieurs tant essentiels que circonstanciels.

2.1 Les compléments circonstanciels (CC) se présentent sous la forme de syntagmes prépositionnels qui situent la relation établie par le substantif noyau et ses actants en décrivant les circonstances de lieu, de temps, etc... dans lesquelles elle se déroule, ou en constituant une limitation, une restriction de cette relation (cf. Tableau $1, \mathrm{~b}$ ). ${ }^{15} \mathrm{La}$ présence des $\mathrm{CC}$, comme celle des circonstants auprès des verbes, n'est pas impliquée par le contenu sémantique du noyau. Il peut par conséquent n'y en avoir aucun, comme il peut y en avoir un nombre illimité (cf. Tesnière 1959: 125). La préposition varie avec la circonstance exprimée:

(15) les ventes sur le territoire français

le décret $n^{\circ} 58-1345$ du 23 décembre 1958 relatif aux agents commerciaux

Quant à l'ordre des expansions actantielles et circonstancielles, il répond dans l'ensemble à ce que faisait déjà observer Tesnière à propos de la phrase, à savoir que les circonstants se placent plutôt après les actants (cf. Tesnière 1959: 127):

(16) $(\mathrm{O}+\mathrm{CC})$ la vente des machines-outils du fabricant sur le territoire français

$\left(\mathrm{A}_{\text {neu }}+\mathrm{CC}\right)$ le prix des marchandises lors de leur achat par le concessionnaire

A cette réserve près que, pour des raisons d'euphonie, de longueur des expansions ou de progression informationnelle dans le texte, l'ordre peut être interverti:

(17) $(\mathrm{CC}+\mathrm{O})$ la vente sur le territoire français des produits visés ci-dessous à l'article 2

Nous intégrerons également dans cette catégorie des compléments circonstanciels les cas un peu spéciaux de structures inversées (cf. 1.2.6). Dans ces structures, comportant un noyau suivi d'un syntagme prépositionnel en $d e$, le terme régissant est

14 Cet exemple nous a été fourni par Hanne Korzen, Ecole des Hautes Etudes Commerciales de Copenhague.

15 Nous incluons également dans cette catégorie les cas de datif libre (ex. 'l'achat des marchandises pour M. Dubois'). 
en fait un complément circonstanciel de lieu ou de temps du terme subordonné - ce que révèle d'ailleurs une inversion de la structure (le complément s'exprimera alors sous forme d'une concrétisation de la formule initiale):

(18) la forêt du crime (le crime de la forêt de Fontainebleau)

le jour/la date de la signature du contrat (la signature du 3.2.1993)

2.2 Les modifieurs essentiels comprennent un nombre limité de syntagmes prépositionnels en de exprimant une durée ou une quantité impliquée par le contenu lexical du noyau (cf. Tableau 1, c). Le caractère essentiel des liens existant entre les deux termes peut s'illustrer en paraphrasant leur relation au moyen d'une phrase de type 'N2 ETRE N1' dans laquelle le verbe dénote un rapport d'identité:

(19) une durée de trois ans [trois ans ETRE une durée]

une remise de 30\% [30\% ETRE une remise]

un total/un montant de 10000 DM [10000 DM ETRE un total/un montant]

Les modifieurs essentiels ne peuvent pas, en règle générale, être séparés de leur noyau par un complément, même si ce dernier est actantiel:

(20) la durée de cinq ans du contrat

Mais non: *la durée du contrat de cinq ans (la construction est seulement possible si de cinq ans qualifie contrat et non durée, et se situe ainsi à un second niveau de profondeur).

2.3 Les modifieurs circonstanciels sont des membres de phrase qui modifient le substantif noyau en lui adjoignant une propriété ou une qualité, mais qui ne sont pas spécifiés par le contenu lexical de ce noyau (cf. Tableau 1. d). Ils se présentent sous forme de syntagmes prépositionnels classificatoires, proches de par leur fonction des épithètes adjectivales. Notre corpus contient les catégories suivantes:

- le type de/à $+\mathbf{N}$, qui ne comporte pas de déterminant. Le degré de cohésion et l'unité sémantique entre $\mathrm{N} 1$ et $\mathrm{N} 2$ sont tels que le syntagme est plutôt à considérer comme un intermédiaire entre le syntagme complexe et le nom composé:

(21) un abus de droit

les frais de transport

le contrat d'agence

une note de service

les prix de vente

une vente à perte ${ }^{16}$

D'éventuelles expansions suivent l'ensemble [N1 prép N2], même lorsqu'il s'agit de compléments actantiels impliqués seulement par le premier des deux substantifs:

16 Emile Benveniste qualifie les SN de ce type de synapsies (1974: $171 \mathrm{ss}$ ); Michael Herslund parle d'incorporations (à paraître). On peut rapprocher des exemples ci-dessus les syntagmes moins fréquents du type après $+\mathbf{N}$ et avec $+\mathbf{N}$ (ex. 'service après vente', 'lettre recommandée avec accusé de réception'). 
(22) (S) la liquidation de biens du concédant

$\left(A_{\text {neu }}\right)$ aucun droit de gage sur ceux-ci

- divers syntagmes prépositionnels sans article défini (à l'exception de l'article défini cataphorique ou générique) exprimant soit une propriété qui qualifie le référent du noyau par rapport à d'autres entités comparables:

(23) des décisions de ce genre

les accidents de toute nature

soit une durée:

(24) une conférence de deux heures

un engagement d'une durée supérieure à un an

soit encore une cause:

(25) la rupture du contrat pour quelque cause que ce soit

ou bien la manière dont quelque chose a lieu:

(26) la rédaction du contrat en langue française

la rédaction du contrat dans la langue de Molière

la vente de tel ou tel produit sous telle ou telle marque

la résiliation du présent contrat aux torts de l'agent, moyennant dommages et intérêts pour la société

Nous venons d'appliquer le schéma de Herslund et Sørensen aux substantifs valentiels et de montrer ainsi le parallélisme existant entre l'analyse de la phrase, avec le verbe comme centre régissant, et l'analyse du syntagme nominal avec le substantif valentiel comme le noeud autour duquel gravitent les autres unités. Il nous reste à présent à voir si un schéma du même type peut expliquer la nature des liens existant au sein de syntagmes à noyaux non valentiels.

\section{Les substantifs non valentiels et leurs expansions}

Les substantifs non valentiels sont, rappelons-le, ceux qui ne reproduisent aucun schéma actantiel. Pourtant, une analyse des relations qu'ils établissent avec leurs expansions révèle qu'on peut les intégrer dans un tableau quadripartite sinon identique du moins parallèle à celui utilisé précédemment, avec deux classes de compléments et deux classes de modifieurs, subdivisées à leur tour suivant que la présence des expansions est déterminée ou non par le contenu sémantique du noyau. Ces quatre classes feront successivement l'objet de notre attention.

\subsection{Les compléments essentiels des substantifs non valentiels: les compléments partitifs.}

Nous rangerons dans ce groupe les syntagmes prépositionnels compléments introduits par de, qui établissent une relation plus ou moins abstraite de la partie au tout. Ces 
compléments partitifs sont pour les substantifs non valentiels ce que les compléments actantiels étaient pour les substantifs valentiels: le rapport entre les deux sortes d'entités est tout aussi étroit en ce que le référent du noyau représente un élément, une composante, une partie du référent de l'expansion et se trouve donc indissolublement lié à celui-ci. On peut dire ici aussi que N2 est impliqué par le contenu lexical de N1. L'étroitesse du lien ressort avec d'autant plus d'évidence qu'on peut paraphraser ces syntagmes à l'aide d'une phrase ayant N2 comme sujet, du type 'N2 AVOIR N1', signifiant que $\mathrm{N} 1$ constitue un sous-ensemble de $\mathrm{N} 2$ et dénotant une relation d'appartenance ou de possession inaliénable:

(27) la moitié du capital de la société [le capital de la société AVOIR une moitié]

dix pour cent du montant des factures [le montant des factures AVOIR dix pour cent]

le dernier jour de chaque mois [chaque mois AVOIR un dernier jour]

les mains/la tête de M. Dubois [M. Dubois AVOIR des mains/une tête]

\subsection{Les compléments circonstanciels des substantifs non valentiels}

Bien qu'à certains égards identiques aux expansions comparables des substantifs valentiels, ils en diffèrent par endroits, comme nous allons le voir en analysant les compléments locatifs et les compléments relationnels.

3.2.1 Les compléments locatifs sont identiques aux syntagmes prépositionnels décrits en 2.1. Etant circonstanciels, ils peuvent figurer aussi bien dans des syntagmes valentiels que dans des syntagmes non valentiels. Ils expriment le lieu, le temps, la restriction, etc... et ici aussi la préposition varie avec la circonstance décrite:

(28) la Cour d'Appel de Nîmes

le train de Paris (cit. Tesnière 1959: 392)

l'agent du secteur du lieu de livraison

une fois par semaine

un mois avant le terme du contrat ${ }^{17}$

3.2.2 Les compléments relationnels sont des syntagmes prépositionnels introduits par de qui, par l'entremise d'un verbe sous-entendu dénotant une idée de possession, de 'disposition' ou de 'création', constituent une sorte de compléments subjectifs. Dans

17 Lors de la discussion qui a suivi ma communication, on m'a fait observer que ce dernier exemple, 'un mois avant le terme du contrat', pouvait avoir plusieurs interprétations possibles. D'abord, le syntagme prépositionnel peut être considéré comme le résultat de la réduction d'une proposition relative ('un mois qui a précédé le terme du contrat'), situant le substantif noyau dans le temps: si le contrat expire le 30 août, il s'agira, par exemple, du mois de juin. Toutefois, selon le contexte, la construction pourra être interprétée également comme étant ou bien un SN suivi d'un syntagme prépositionnel distinct ('elle a travaillé [un mois] [avant le terme du contrat]') ou bien, le plus souvent, comme un syntagme prépositionnel, dans lequel un mois est une sorte de modifieur qui exprime la durée de la relation d'antériorité en indiquant le moment précis où un événement a eu lieu ('elle a téléphoné [un mois avant le terme du contrat]'). Voir à ce sujet Vikner (1990). 
certains cas, le SN n'aura qu'une seule lecture possible, mais le plus souvent, ce n'est qu'en consultant le contexte qu'on pourra le placer dans l'une des trois catégories suivantes.

- Les compléments de possession traduisent, comme leur nom l'indique, un rapport de possession ou de propriété:

(29) le magasin de M. Rabut (M. Rabut possède le magasin)

l'enseigne $d u$ concédant (le concédant possède l'enseigne)

le capital de la société (la société possède le capital)

- Les compléments de 'disposition' expriment que, d'une manière ou d'une autre, on 'dispose' de quelque chose:

(30) le fauteuil $d$ 'Edouard (Edouard dispose du fauteuil)

le pays/la ville de l'agent (l'agent habite le pays/la ville)

le secteur de l'agent (l'agent est responsable du secteur)

le garage de la voiture (la voiture se gare dans le garage)

- Les compléments de 'création' représentent une idée de création, de fabrication, de construction:

(31) la banque/les machines de la société (la société a créé la banque/les machines)

les porcelaines de la manufacture de Sèvres (la manufacture de Sèvres a fabriqué les porcelaines)

la maison $d u$ concessionnaire (le concessionnaire a construit la maison)

Dans ce dernier cas, la relation entre N2 et N1 pourrait correspondre aussi bien à un rapport possessif ('le concessionnaire possède la maison') qu'à un complément de disposition ('le concessionnaire est locataire de la maison'). Seul le contexte textuel et/ou extra-textuel permettrait de trancher à ce sujet.

3.3 Les modifieurs essentiels des substantifs non valentiels comprennent, comme le faisaient les expansions équivalentes des syntagmes valentiels (cf. 2.2), un nombre limité de constructions prépositionnelles quantitatives introduites par de et impliquées par le contenu lexical du noyau. De même que précédemment, nous les analyserons comme une sorte d'expansions subjectives dans une construction pouvant être paraphrasée par 'N2 ETRE N1':

(32) une période de dix semaines [dix semaines ETRE une période]

3.4 Les modifieurs circonstanciels des substantifs non valentiels sont, ici également, des syntagmes prépositionnels classificatoires qui précisent une propriété ou une qualité du noyau sans être impliqués pour autant par le contenu lexical de celui-ci. Les occurrences relevées dans notre corpus montrent des catégories comparables à celles rencontrées plus haut (cf. 2.3):

- le type de $+\mathbf{N}$ (subsidiairement à $+\mathbf{N}$ : machine à coudre) ne comportant pas de déterminant et formant une unité sémantique avec le noyau qui rapproche la construction du nom composé: 
(33) les pièces de rechange

la Cour d'Appel

le lieu d'arbitrage

l'agent de commerce

son champ d'activité

le bon de garantie

le chiffre d'affaires annuel

un homme de génie / une femme de tête (cit. Tesnière 1959: 391)

A cela s'ajoute un petit groupe de structures définitoires diverses, rares dans les textes juridiques, où c'est le premier élément qui qualifie le second (Tesnière, dans une optique différente, désigne les expansions de ces structures sous le nom d'adjectifs de quiddité, 1959: $445 \mathrm{ss}$ ). Le substantif régissant peut être affectivement indifférent et sera alors précédé de l'article défini ((34) a.). Mais il peut aussi avoir une valeur affective hypocoristique ((34) b.) ou même, la plupart du temps, clairement péjorative ((34) c. et d.) et s'emploiera alors avec un article indéfini, un adjectif possessif ou un adjectif démonstratif:

(34) a. la ville de Nîmes

b. un trésor d'enfant

c. mon imbécile de frère

d. ce filou d'Edouard

- divers syntagmes prépositionnels sans article défini (à l'exception de l'article défini cataphorique ou générique) exprimant une propriété ou une qualité caractéristique de $\mathrm{N} 1$. Les syntagmes prépositionels peuvent avoir différentes formes, comme par exemple de + article indéfini + adjectiflocution adjectivale $+N$ :

(35) un homme d'une grande beauté

une chaise $d^{\prime}$ 'un bois exotique

des machines-outils d'une autre marque que celle de la société Outima

de + adjectif possessif $+N$, où le possessif reproduit un actant du nom valentiel qu'il précède:

(36) une personne de son choix

de + article générique $+N$ :

(37) la semaine du livre

l'année de la femme

\section{Conclusion}

L'analyse à laquelle nous nous sommes consacrée montre qu'aux quatre catégories initiales d'expansions pouvant caractériser les substantifs valentiels, et répondant au schéma quadripartite de Herslund et Sørensen, correspondent quatre autres catégories 
lorsque les noyaux sont non valentiels. Les SN se laissent donc répartir en huit classes, ainsi que l'illustre le tableau suivant:

\begin{tabular}{|c|c|c|}
\hline & Expansions essentielles & Expansions circonstancielles \\
\hline \multirow[t]{2}{*}{$\begin{array}{l}\text { Substantifs } \\
\text { valentiels }\end{array}$} & $\begin{array}{l}\text { a. Compléments actantiels } \\
\text { (S) l'expiration du contrat } \\
\text { (O) la livraison des marchandises } \\
\left(\mathrm{A}_{\text {neu }}\right) \text { son consentement à la succession } \\
\left(\mathrm{A}_{\text {dat }}\right) \text { la vente des produits à la clientèle } \\
\text { (Aloc) une meilleure pénétration sur le } \\
\text { marché danois } \\
\text { Structures inversées } \\
\text { (S) l'homme de la russification } \\
\text { (O) la Pologne de l'invasion } \\
\text { (Adat) les enfants de la promesse }\end{array}$ & $\begin{array}{l}\text { la vente des machines-outils sur le } \\
\text { territoire français } \\
\text { le décret du } 23 \text { décembre } 1958 \\
\text { Structures inversées } \\
\text { la forêt du crime } \\
\text { le jour de la signature du contrat }\end{array}$ \\
\hline & $\begin{array}{l}\text { c. Modifieurs essentiels } \\
\text { une durée de trois ans }\end{array}$ & $\begin{array}{l}\text { d. Modifieurs circonstanciels } \\
\text { un abus de droit } \\
\text { les frais de transport } \\
\text { des décisions de ce genre } \\
\text { une conférence de deux heures } \\
\text { la rédaction du contrat en langue } \\
\text { française }\end{array}$ \\
\hline \multirow[t]{2}{*}{$\begin{array}{l}\text { Substantifs } \\
\text { non } \\
\text { valentiels }\end{array}$} & $\begin{array}{l}\text { a'. Compléments essentiels: } \\
\text { les compléments partitifs } \\
\text { la moitié du capital de la société } \\
\text { le dernier jour de chaque mois }\end{array}$ & $\begin{array}{l}\text { b'. Compléments circonstanciels } \\
\text { 1) Compléments locatifs } \\
\text { la Cour d'Appel de Nîmes } \\
\text { 2) Compléments relationnels } \\
\text { (possession) le magasin de M. Rabut } \\
\text { ('disposition') le fauteuil d'Edouard } \\
\text { ('création') les porcelaines de la } \\
\text { manufacture de Sèvres }\end{array}$ \\
\hline & $\begin{array}{l}\text { c'. Modifieurs essentiels } \\
\text { une période de dix semaines }\end{array}$ & $\begin{array}{l}\text { d'. Modifieurs circonstanciels } \\
\text { les pièces de rechange } \\
\text { la Cour d'Appel } \\
\text { un homme d'une grande beauté } \\
\text { une personne de son choix } \\
\text { la semaine du livre }\end{array}$ \\
\hline
\end{tabular}

Tableau 2

Seules les expansions de la catégorie a. sont actantielles. Comme elles sont très fréquentes et qu'elles peuvent se combiner avec d'autres expansions pouvant à leur tour 
reproduire une valence verbale, les syntagmes à noyau valentiel auront virtuellement le plus grand nombre d'expansions.

Tout substantif valentiel implique, par son contenu même, un certain nombre d'actants qui existeront à l'état latent jusqu'à ce qu'ils soient actualisés dans un complément essentiel. Ainsi la valence nominale est une virtualité dont l'actualisation dépend de la présence d'une expansion actantielle. Il s'est révélé par ailleurs que des rapports verbaux non valentiels pouvaient s'établir entre un substantif et une de ses expansions par l'entremise d'un verbe sous-entendu. Ce verbe sera typiquement ETRE avec les modifieurs essentiels, AVOIR avec les compléments partitifs, et un verbe dénotant une idée de possession, de 'disposition' ou de 'création' lorsqu'il s'agira de préciser la nature des liens sémantiques unissant un substantif non valentiel et ses compléments relationnels. Or, ces expansions 'verbales' non actantielles ont en commun de ne pouvoir être que subjectives. Nous conclurons par conséquent en établissant que si les diverses variétés de $\mathrm{SN}$ renfermant des expansions verbales correspondent à différents types de propositions libres, la nature des expansions se réduit, sur le plan logico-sémantique aux relations suivantes:

- prédicat nominal et sujet, objet ou adjet lorsque le rapport est valentiel,

- prédicat sous-entendu et sujet lorsque le rapport est non valentiel.

On voit dès lors que la valence nominale est une dimension verbale parmi d'autres pouvant exister à l'intérieur d'un syntagme nominal, mais elle est la seule qui, lorsqu'elle est actualisée, est en mesure de traduire la multitude des relations pouvant être exprimées dans la proposition libre par le verbe et les diverses unités qui gravitent autour de lui.

\section{Bibliographie}

Baron, Irène (1992). 'Les syntagmes nominaux complexes dans les textes juridiques français'. HERMES, Journal of Linguistics 9, 19-42.

Baron, Irène (à paraître). 'Complex Noun Phrases in Danish. A Valence Perspective' (Université de Odense).

Bartning, Inge (1992). 'La préposition de et les interprétations possibles des syntagmes nominaux complexes. Essai d'approche cognitive'. Lexique 11, 163-191.

Benveniste, Emile (1966). 'Pour l'analyse des fonctions casuelles: le génitif latin'. Problèmes de linguistique générale. Paris: Gallimard, 139-148.

Benveniste, Emile (1974). 'Formes nouvelles de la composition nominale'. Problèmes de linguistique générale 2. Paris: Gallimard, 163-176.

Comrie, Bernard / Sandra A. Thompson (1985). 'Lexical nominalization'. T. Shopen, éd.: Language Typology and Syntactic Description. Vol. III. Grammatical Categories and the Lexicon. Cambridge University Press, 349-398. 
Cornu, Gérard (1990). Linguistique juridique. Paris: Montchrestien.

Herslund, Michael (1982). 'Ergative Substructures in 'Objective' Languages ?' $T$. Fretheim / L. Hellan, éds.: Papers from the Sixth Scandinavian Conference of Linguistics. Trondheim, 75-83.

Herslund, Michael (1988a). Le datif en français. Editions Peeters, Louvain-Paris.

Herslund, Michael (1988b). 'On Valence and Grammatical Relations'. F. Sørensen, éd.: Valency. Three Studies on the Linking Power of Verbs. Copenhagen Studies in Language, CEBAL Series 11. Copenhague: Nyt Nordisk Forlag, 3-34.

Herslund, Michael (à paraître). 'La notion d'incorporation en danois et en français' (Université de Strasbourg).

Herslund, Michael / Finn Sørensen (1985). De franske verber. En valensgrammatisk fremstilling. I. Verbernes syntaks. Institut d'Etudes Romanes, Université de Copenhague ( $2 \mathrm{e}$ édition: 1990, Samfundslitteratur).

Robert, Paul (1991). Le Petit Robert 1. Paris: Le Robert.

Sourioux, Jean-Louis / Pierre Lerat (1975). Le langage du droit. Paris: PUF.

Spang-Hanssen, Ebbe (1963). Les prépositions incolores du français moderne. Copenhague: Gad.

Stage, Lilian (1986). 'Franske substantivers valens'. N. Davidsen-Nielsen / F. Sørensen, éds.: Festskrift til Jens Rasmussen. CEBAL 8. Copenhague: Nyt Nordisk Forlag, 204-229.

Stage, Lilian (à paraître). 'La valence des noms en français. Etude sur le rôle du syntagme prépositionnel dans les syntagmes nominaux complexes'. M. Herslund, éd.: NP Structures. Copenhagen Studies in Language 17.

Tesnière, Lucien (1959). Eléments de syntaxe structurale. Paris: Klincksieck $\left(2^{\mathrm{e}}\right.$ édition, $5^{\mathrm{e}}$ tirage: 1988$)$.

Ulland, Harald (1991). Les nominalisations agentive et instrumentale en français moderne. Bergen, Thèse.

Vikner, Carl (1990). 'Aspekt og tidsreference ved inden og efter'. ARK 54. Ecole des Hautes Etudes Commerciales de Copenhague, 43-70.

Wiberg, Lars (1956). 'Etude sur les expressions du type 'la fondation de Rome par Romulus". Studia Neophilologica XXVIII, 148-217.

Povzetek

SAMOSTALNIŠKA BESEDNA ZVEZA V FRANCOŠČINI GLEDE NA VEZLJIVOST

Prispevek obravnava samostalniške besedne zveze $v$ francoščini glede na teorijo o glagolski vezljivosti.

Samostalniške besedne zveze so pretvorba prostega stavka $v$ samostalniško obliko in torej prevzemajo razvrstitveno shemo, značilno za stavek. Podoba te sheme je odvisna od tega, ali odnosnica glagolsko vezljivost odraža (vezljivostni samostalniki) ali ne (nevezljivostni samostalniki). Tema dvema vrstama samostalnikov se lahkko pridružijo različna določila. Prispevek predlaga razvrstitev samostalniških besednih zvez v osem različnih skupin. Opis posameznih razredov omogoča določitev odnosov znotraj besednih zvez in s tem pojma vezljivosti $v$ tem kontekstu. 